\title{
Comparison of Visual Acuity for Different Amount of Deviation of Alternate Exotropia
}

\section{Partha Haradhan Chowdhury ${ }^{*}$ and Brinda Haren Shah ${ }^{2}$}

1M. Optom, Department of Optometry, Shree Satchandi Jankalyan Samiti Netra Prasikshan Sansthan, Pauri, Affiliated to Uttarakhand State Medical Faculty, Dehradun, India

${ }^{2}$ M. Optom, Department of Optometry, Gujarat University, Gujarat, India

\section{Research Article}

Volume 3 Special Issue 1

Received Date: July 30, 2018

Published Date: August 15, 2018

*Corresponding author: Partha Haradhan Chowdhury, M. Optom, Department of Optometry, Shree Satchandi Jankalyan Samiti Netra Prasikshan Sansthan, Pauri, Affiliated to Uttarakhand State Medical Faculty, Dehradun, India, Email: optometrypublish@gmail.com

\section{Abstract}

Methods: A pilot, cross sectional, observational study was performed at tertiary eye care centers. Subjects with Ocular deviation between 10 to 40 prism diopters, Corrected distance Visual Acuity should be greater than 6/18 and Age should be between 10 to 40 years of age were included in the study. Visual Acuity was assessed with Log Mar chart.

Results: 30 subjects were included in the study. Out of that, 16 subjects were in the age group of 11-20 years, 12 subjects were in the age group of 21-30 years and 2 subjects were in the age group of 31-40 years. $60 \%$ subjects were Female and $40 \%$ subjects were Male. The mean visual acuity was considered in each amount of deviation. It shows that maximum acuity is deteriorated in the ocular deviation of 31-40 prism diopters.

Conclusions: As the amount of ocular deviation increases, visual acuity will be more deteriorated.

Keywords: Visual Acuity; Alternate Esotropia; Alternate Exotropia

\section{Introduction}

In case of ocular deviation, images of an object fall on the parafoveal region. In case of Esodeviation, the images of an object fall on the nasal foveal region and in case of Exodeviation, the images of an object falls on the temporal parafoveal region. In the macular region, anatomical consideration is a very important factor because of density and number of photoreceptor cells. In the foveal region, the number of cone cells is highest compared to parafoveal region and the density of cone cells are highest in the foveal region as compared to para foveal region. Cone cells are solely responsible for visual acuity. If density of the cone cells are deteriorated due to any reason, then there may be lots of chances for deterioration of visual acuity. In cases of alternate exotropia, the images are focused on parafoveal region alternately and in case of considerable amount of alternate exotropia, there may be chances of deterioration of visual acuity.

\section{Methodology}

Cross sectional, pilot and observational study was performed at tertiary eye care centers. Inclusion criteria includes Subjects with Ocular deviation between 10 to 40 
prism diopters, Corrected distance Visual Acuity should be greater than 6/18 and Age should be between 10 to 40 years of age. Individuals with any other systemic disease(specially which can affect study), Individuals with any other Ocular Pathology, with any active ocular infection, any ocular anomalies like Corneal Scar etc ,ocular deviation if less than 10 degree and Significant amount of amblyopic patient were excluded from the study. Full refractive correction along with detailed fundus evaluation was performed in each and every patient. Visual Acuity was assessed with Log Mar Chart in different amounts of deviation of Alternate Exotropia. Data analysis was done using SPSS software version 20 [1-3].

\section{Results}

A total of 30 subjects were included in the study. Age wise distribution is shown in figure 1. 16, 12 and 2 subjects were in the age group of 11-20 years, 12 21-30 years and 2 subjects were in the age group of 31-40 years. Figure 2 shows gender wise distribution of the subjects. $60 \%$ subjects were Female and $40 \%$ subjects were Male. Figure 3 shows comparison of mean Visual Acuity of subjects with Alternate Exotropia with different amount of ocular deviation. In Alternate Exotropia of 11-20 prism diopters, mean Visual Acuity is 0.09 log units. In Alternate Exotropia of 21-30 prism diopters, mean Visual Acuity is $0.13 \mathrm{log}$ units. In Alternate Exotropia of 31-40 prism diopters, mean Visual Acuity is 0.18 log units. This shows that maximum acuity is deteriorated in the ocular deviation of 31-40 prisms.

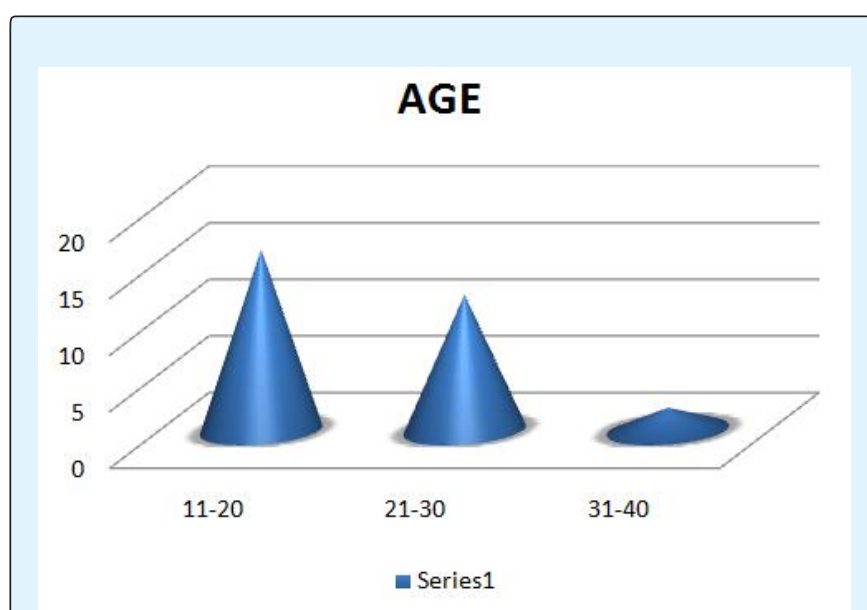

Figure 1: Shows Age wise Distribution of the Subject.


Figure 3: Shows comparison of Contrast Sensitivity for different amount of deviation for Exotropia.

\begin{tabular}{|c|c|}
\hline Visual Acuity & Log Units \\
\hline $11-20$ & 0.09 \\
\hline $21-30$ & 0.13 \\
\hline $31-40$ & 0.18 \\
\hline
\end{tabular}

Table 1: Acuity Is Deteriorated.

\section{Discussion}

Ocular deviation is a very important factor for other parameters like contrast sensitivity, colour vision and for visual acuity also. Ocular deviation is an important factor because due to anatomical consideration of the macular
Partha Haradhan Chowdhury and Brinda Haren Shah. Comparison of Visual Acuity for Different Amount of Deviation of Alternate Exotropia. J Ophthalmol 2018, 3(S1): 000S1-007. 


\section{Open Access Journal of Ophthalmology}

area. In the foveal area, cone density is highest compared to para foveal region. In case of Exo deviation, the image is shifted to temporal region of the para fovea. In case of Alternate Exodeviation the images are shifted to temporal para foveal region alternately. That's why; at the temporal region cone cell density is reduced compared to foveal region. So, in case of Alternate Exodeviation, deterioration of visual acuity is being observed [4-7].

\section{Conclusion}

As the amount of ocular deviation increases, visual acuity will be more deteriorated.

\section{References}

1. Kenneth $\mathrm{W}$ Wright, Peter H Spiegel, Lisa Thompson (2006) Handbook of Pediatric Strabismus and Amblyopia. 1st (Edn.).

2. Hui Zhu, Jia Jia Yu, Rong Bin Yu, Hui Ding, Jing Bai, et al. (2015) Association between Childhood Strabismus and Refractive Error in Chinese Preschool Children. Plos One 10(6): e0130914.
3. Zhale Rajavi, Hamideh Sabbaghi, Ahmad Shojaei Baghini, Mehdi Yaseri, Koroush Sheibani, et al. (2015) Prevalence of Colour Vision Deficiency and its Correlation with Amblyopia and Refractive Errors among Primary School Children. J Ophthalmic Vis Res 10(2): 130-138.

4. Anika K Tandon, Velez FG, Isenberg SJ, Demer JL, Pineles SL (2014) Binocular Inhibition in Strabismic Patients is Associated with Diminished Quality of Life. JAAPOS 18(5): 423-426.

5. Ye XC, Pegado V, Patel MS, Wasserman WW (2014) Strabismus genetics across a spectrum of eye misalignment disorders. Clin Genet 86(2): 103-111.

6. Kocak-Altintas AG (2000) Colour vision and Colour Vision deficiency in Amblyopia. European Journal of Ophthalmology 10(1): 77-81.

7. Alan W Freeman (1996) Components of Colour vision Loss in Strabismus. Journal of vision research 36(5): 765-774.

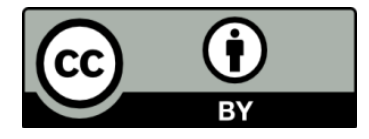

